\title{
J Is for JavaScript: \\ A Direct-Style Correspondence \\ between Algol-Like Languages and JavaScript Using First-Class Continuations
}

\author{
Olivier Danvy ${ }^{1}$, Chung-chieh Shan $^{2}$, and Ian Zerny ${ }^{1}$ \\ ${ }^{1}$ Department of Computer Science, Aarhus University \\ Aabogade 34, DK-8200 Aarhus N, Denmark \\ $\{$ danvy, zerny\}@cs.au.dk \\ ${ }^{2}$ Department of Computer Science, Rutgers University \\ 110 Frelinghuysen Road, Piscataway, NJ 08854, USA \\ ccshan@rutgers.edu
}

\begin{abstract}
It is a time-honored fashion to implement a domain-specific language (DSL) by translation to a general-purpose language. Such an implementation is more portable, but an unidiomatic translation jeopardizes performance because, in practice, language implementations favor the common cases. This tension arises especially when the domain calls for complex control structures. We illustrate this tension by revisiting Landin's original correspondence between Algol and Church's lambdanotation.

We translate domain-specific programs with lexically scoped jumps to JavaScript. Our translation produces the same block structure and binding structure as in the source program, à la Abdali. The target code uses a control operator in direct style, à la Landin. In fact, the control operator used is almost Landin's J-hence our title. Our translation thus complements a continuation-passing translation à la Steele. These two extreme translations require JavaScript implementations to cater either for first-class continuations, as Rhino does, or for proper tail recursion. Less extreme translations should emit more idiomatic control-flow instructions such as for, break, and throw.

The present experiment leads us to conclude that translations should preserve not just the data structures and the block structure of a source program, but also its control structure. We thus identify a new class of use cases for control structures in JavaScript, namely the idiomatic translation of control structures from DSLs.
\end{abstract}

\section{Introduction}

It has long been routine to define a programming language by writing an interpreter in or a compiler towards a pre-existing language [27, 28, 32, 39, 40, 41, This tradition began with John McCarthy 34, who argued that it is not a circular argument but a valid pedagogical device to use a pre-existing language as a 
notation for expressing computation. McCarthy drew an analogy between translations from one programming language to another and Tarski's efforts to define one mathematical logic in terms of another, "which have proved so successful and fruitful" [34, page 7] even though they still draw criticism today [19].

Translations between programming languages have also been used to reason about expressiveness. For example, Böhm and Jacopini used a compiling argument to show that flowcharts and expression languages with recursion have the same expressive power [7, and Fischer invented the CPS transformation to show the equivalence between the deletion strategy and the retention strategy to implement activation records 16. Finally, this style of formal specification is also useful for building interpreters and compilers in practice. Indeed, it has given rise to industrial-strength software development [6].

The translation approach is alive and well today, whether the defined (source) language is considered to be domain-specific or general-purpose, and whether the defining (target) language is a variant of the lambda calculus, C, or some other language. This stream of successes is especially remarkable given that differences between the defined and defining languages often force the translation to be quite ingenious, albeit not entirely perspicuous.

Pervasive ingenuity: Some translations impose global changes on programs. For example, when the defined language features a side effect that the defining language does not support, a definitional interpreter must encode the effect throughout the translation using, e.g., state-passing or continuationpassing style 41]. These styles have since been crisply factored out into computational monads 38,49.

Homomorphic ingenuity: Some translations manage to relate structural elements between source and target programs. For example, McCarthy translated flowcharts to mutually recursive equations by mapping each program point to a recursive equation and each variable to a parameter in a recursive equation 33. These equations were tail-recursive, a property that Mazurkiewicz then used for proving properties about flowcharts [31. Landin translated Algol programs to applicative expressions by mapping block structure to subexpression structure, assignments to state effects, and jumps to control effects [27]. Working homomorphically with a smaller defining language, namely the lambda calculus, Abdali mapped local functions into global, lambda-lifted recursive equations in a storeless fashion [1, 3], but invented continuation-passing style en passant to implement jumps [2].

Seemingly no ingenuity: Even when the translation turns out to be just an identity function, careful design may be required. For example, it is desirable for a partial evaluator to be Jones optimal, which means that specializing a self-interpreter to a program yields the same program modulo renaming [21. Achieving Jones optimality requires much of the partial evaluator (e.g., it must resolve scoping references statically), but also of the interpreter: for one thing, the interpreter must be in direct style; indeed, specializing a continuation-passing self-interpreter with a Jones-optimal partial evaluator yields programs in continuation-passing style. To take another example, even 
though Pascal can be regarded as a subset of Scheme and hence easily translated to Scheme by (essentially) the identity function, such a translation calls for a Scheme compiler, such as Orbit 25], that is designed so that Pascallike programs (i.e., programs with only downwards funargs 1 and downwards contargs) would be compiled as efficiently as by a Pascal compiler 26.

Shoehorning: Not all properties of the defined language are naturally supported by the defining language and its run-time system. Perhaps the best-known example is proper tail-recursion and its implementation by trampolining [4, 18, 37, 43, 47. A close second is first-class continuations [30, 36, 44].

For humans and machines to work with a translation more easily, it is our belief that homomorphic ingenuity and seemingly no ingenuity are preferable over pervasive ingenuity and shoehorning. In short, we believe that a translation should take advantage of the expressive power of the defining language in an idiomatic way, if only for implementations of the defining language to execute target programs more efficiently. As shown by the examples above, this goal of idiomaticity often calls for the defined and defining languages to be carefully designed and reconciled. In other words, the principle of idiomaticity guides not just language implementation but also language design, especially today as the proverbial 700 DSLs blossom on new platforms such as browsers running JavaScript.

This article establishes an idiomatic translation between a specific and illustrative pair of languages.

- Our defined language is an Algol-like block-structured language with lexically scoped jumps. It is illustrative not just because most DSLs have block structure and lexical scope, but also because many DSLs feature complex control constructs motivated by their domains such as pattern matching and logical inference.

- Our defining language is JavaScript with first-class continuation objects, as implemented in Rhino [10. It is illustrative not just because many DSLs are implemented by translation to JavaScript, but also because it lets us exhibit an extremely uniform and frugal translation among more complex alternatives that use a greater variety of control-flow instructions.

Taking advantage of the close correspondence between Rhino's continuation objects and Landin's J operator [14], we thus revive Landin's original correspondence between Algol programs and applicative expressions [27,28].

\footnotetext{
1 'Funarg' is an abbreviation for 'functional argument' and by analogy, 'contarg' stands for 'continuation argument'. They refer to the ability of passing functions as arguments (downwards funargs) and returning functions as results (upwards funargs). The 'downward' and 'upward' orientation stems from implementing function calls with a current control stack: downwards is toward what has been pushed (and is still there) and upwards is toward what has been popped (and thus may be gone [16]).
} 


\section{Continuation Objects in JavaScript}

This section introduces continuation objects in the JavaScript implementation Rhino, compares them to Landin's J operator, and explains how we use them to express jumps in a block-structured language.

In Rhino, a continuation object can be created by evaluating the expression new Continuation(). The resulting object represents the continuation of the call to the function that evaluated this expression. That is, invoking a continuation object (as if it were a function) returns from the function that created it. For example, the following program only prints 2 .

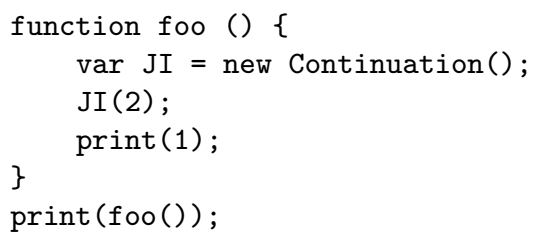

The continuation is undelimited and remains valid throughout the rest of the program's execution. For example, the following program prints 1 followed by an unbounded series of 2's.

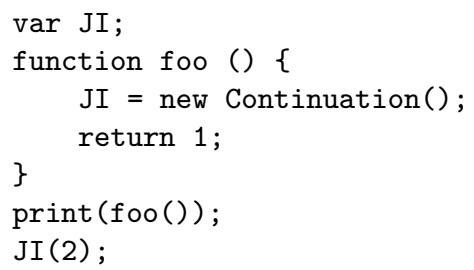

\subsection{Landin's Translation of Jumps Using J}

We name the captured continuations above JI because creating a continuation object in Rhino is equivalent to invoking Landin's J operator on the identity function [14]. The J operator is the first control operator to have graced expression-oriented programming languages. Landin invented it specifically to translate Algol jumps to applicative expressions in direct style [27,29]. If JavaScript featured the $\mathrm{J}$ operator, then Landin would have translated the loop

$$
i:=1000000 \text {; }
$$

loop: $i:=i-1$;

if $i>0$ then goto loop;

to the following JavaScript program.

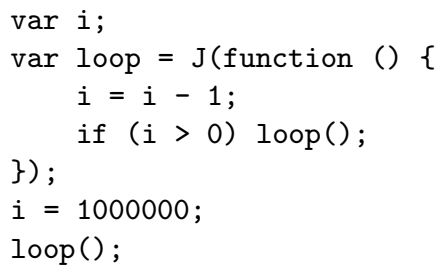


The application of $\mathrm{J}$ above creates a function loop that, when invoked, evaluates the function body $i=i-1$; if ( $i>0$ ) loop(); with the continuation of the call to the program. In other words, loop denotes a "state appender" 8 and the invocation loop() jumps into the function body in such a fashion that the function body directly returns to the caller of the translated program. We thus express a jump to loop as loop(). This program contains two jumps to loop, an implicit fall-through and an explicit goto, so the JavaScript code above contains two occurrences of loop().

The expression new Continuation() in Rhino is equivalent to the expression $\mathrm{J}$ (function ( $\mathrm{x}$ ) \{ return $\mathrm{x} ;$ \}) in JavaScript with the J operator. Conversely, Landin and Thielecke [4] noted that $\mathrm{J}$ can be expressed in terms of JI as

$$
\mathrm{J}=(\lambda c \cdot \lambda f \cdot \lambda x \cdot c(f x)) \mathrm{JI} .
$$

Following Landin's translation strategy, then, one might define

function compose $(c, f)$ return function $(x)\{c(f(x)) ;\}\}$

in JavaScript and then translate the loop above as follows.

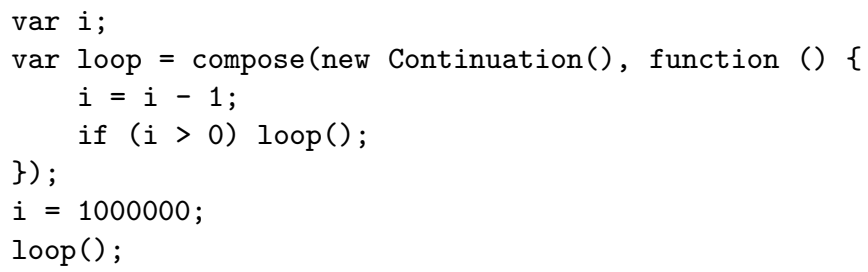

(Because we translate each label to a function that takes no argument, the two occurrences of $\mathrm{x}$ in the definition of compose can be omitted.) This translation, like Landin's, is attractive in that it idiomatically preserves the block and binding structure of the source program: the main program block translates to the main program block, a sequence of commands translates to a sequence of commands, and the variable $i$ and the label loop translate to the variables $i$ and loop.

Unfortunately, although this last translation runs in Rhino, it overflows the stack. The reason is that 1000000 calls to c pile up on the stack and are not discarded until the function body returns for the first time. Although we can express $\mathrm{J}$ in terms of JI, it is unclear how to do so without this space leak.

\subsection{Our Translation of Jumps Using JI}

In order to translate jumps while preserving the stack-space usage behavior of programs, we modify Landin's translation slightly: every captured continuation shall accept a thunk and invoke it immediately [15]. In other words, every function call shall expect a thunk to be returned and invoke it immediately.

Because we cannot change the continuation with which Rhino invokes the main program, this modification requires that we wrap up the main program in a function main, which returns a thunk that should be invoked immediately. Below is our final translation of the loop, which completes without overflowing the stack in Rhino. 


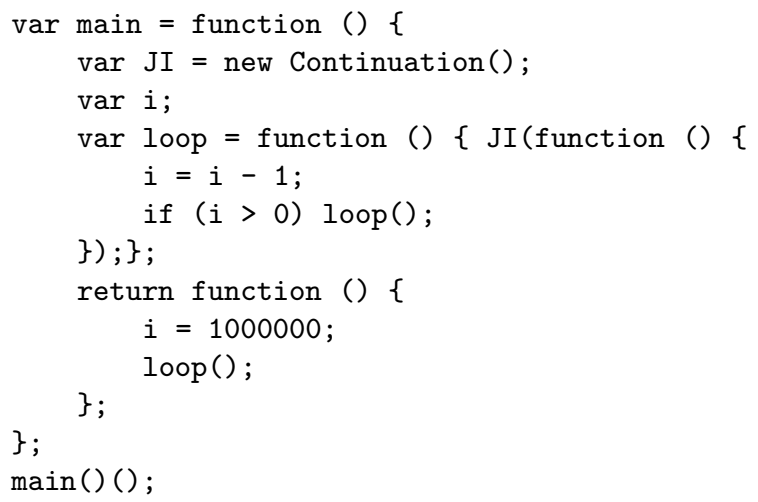

This use of thunks is reminiscent of trampolining, but our technique using JI does not require the caller of a function to invoke thunks repeatedly until a final result is reached. Rather, the continuation of main() accepts a thunk and invokes it exactly once. If another thunk needs to be invoked, such as function () $\{i=i-1$; if $(i>0) \operatorname{loop}()$; $\}$ in this example, the same continuation needs to be invoked again. In other words, the function main in our target program returns exactly once more than the number of times a jump occurs in the source program.

\subsection{Other Uses of J}

Outside of its inventor and his student 8], J was first used by Rod Burstall to traverse a search tree in direct style and breadth first, using a queue of first-class continuations 9]. We have re-expressed Burstall's breadth-first tree traversal in Rhino. With its queue of first-class continuations, this program falls out of the range of our translator from pidgin Algol to JavaScript.

\section{$3 \quad$ Source and Target Languages}

The key property of our direct-style translation is that it is homomorphic and thus preserves idioms: declarations translate to declarations, blocks to blocks, commands to commands, function calls to function calls, and so forth. Since the translation is homomorphic, we dispense with it altogether in this presentation and simply consider the restricted language obtained as the image of the translation. We thus present the grammar of JavaScript programs in the image of the translation. Figure 1 displays the productions of special interest to our translation, accounting for the essential features of Algol we wish translated to JavaScript. For completeness we include the full grammar in Appendix A. This full grammar accounts for all of the example programs (see Section 4).

- A program is a sequence of declarations and commands. Such a program is translated to a top-level function in the same way procedures are, as shown by the <program> production. This translation is required to support labels and jumps at the top level of a program, as illustrated in Section 2.2 . 


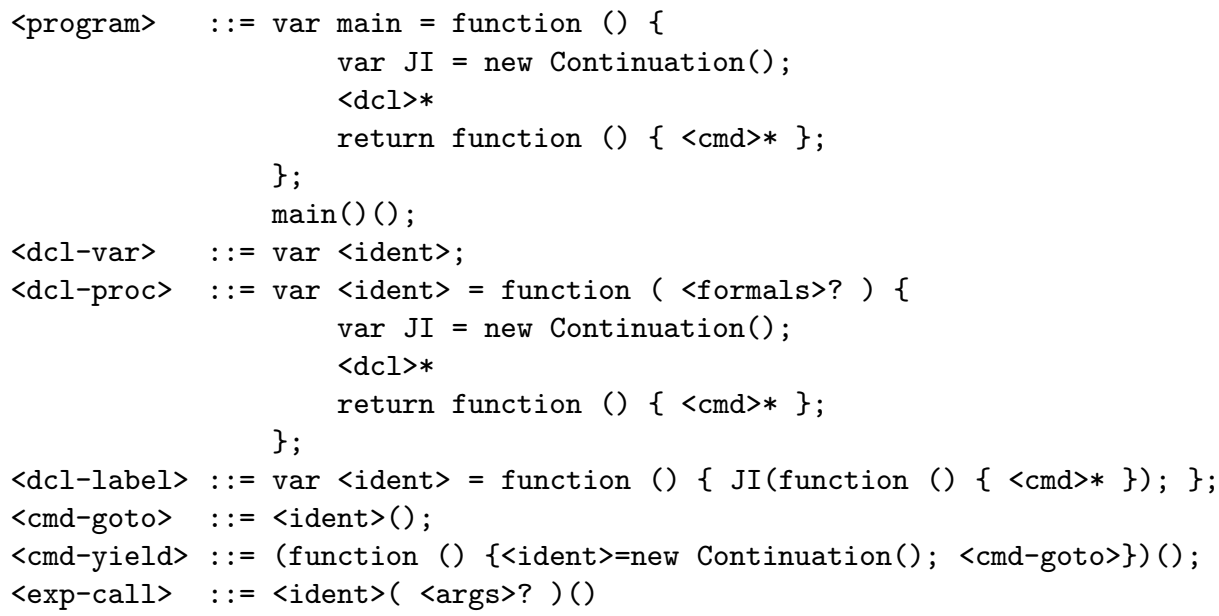

Fig. 1. Essential grammar of JavaScript in the image of the translation

- The <dcl-var > production shows that each variable declaration, is translated directly to a variable declaration in JavaScript. All variables are implicitly initialized to $\perp$, i.e., undefined in JavaScript.

- The <dcl-proc $>$ production shows that a procedure declaration is translated to a JavaScript function that accepts the same formals and returns a thunk containing the procedure commands. In a scope visible to the thunk of commands, nested declarations are defined and the return continuation of the function closure is captured and bound to JI. All declarations are mutually recursive so that declarations can refer to each other as well as JI.

- A label consists of a name and a sequence of commands. For simplicity we assume that explicit jumps have been inserted in the program wherever execution may flow from one label to another. The <dcl-label> production shows that a label definition is translated to a JavaScript function of no arguments, whose body applies the return continuation JI to the thunk of label commands. Thus, invoking this function returns the thunk from the currently running procedure.

- The <cmd-goto> production shows that goto commands are translated to ordinary function calls with no arguments in JavaScript.

- The <cmd-yield> production shows how a yield command consists of rebinding the caller's label with the current continuation, followed by a goto command to transfer control. The surrounding function declaration is required to capture the current continuation. The translation must supply both the from and to labels. The labels are required to be declared in the exact same block. The yield command, however, may appear elsewhere.

- The <exp-call> production shows that a procedure call is translated to a JavaScript function call with the same arguments, and the result of the 
application, a thunk of procedure commands, is forced to obtain the actual result of the procedure call.

All remaining productions, found in Appendix A, show the straightforward and idiomatic translation to JavaScript.

For simplicity we consider only characters in the ASCII character set with some restrictions on their use. All identifiers are required to be alpha-numeric; the set of label identifiers must not overlap with that of variable and procedure identifiers; and $\mathrm{JI}$ is a reserved keyword and must not be used as an identifier. To declare named functions, we use variable binding and function expressions in the form of "var <ident> = function ...". This is necessary as function declarations can, in our translation, appear in any block statement, whereas in JavaScript function declarations are only valid at the top-level or directly inside a function body.

\section{A Representative Collection of Program Samples}

The goal of this section is to illustrate with classical examples the direct-style correspondence between pidgin Algol with lexically scoped jumps and JavaScript with continuation objects. We consider in turn backward jumps within the same block (Section 4.1), backward and forward jumps within the same block (Section 4.2), outwards jumps (Section 4.3), and coroutines (Section 4.4). For what it is worth, our embedding passes Knuth's man-or-boy test (Section 4.5).

Each of the following kinds of jumps, except for coroutines, can be translated as a special case using more specialized control structures offered by JavaScript. We discuss such specialized translation schemes further in Section 5 .

\subsection{Backward Jumps}

To simulate backward jumps within the same block, our translation simply declares a sequence of lexical variables, each denoting the continuation of a label, and uses one of these variables for each jump.

Backward jumps can be used to repeatedly try a computation until a condition is met, as in a loop. It can also be used to express more involved iteration patterns that recur in domains such as pattern matching and logical inference. These patterns are exemplified by the search phase of Knuth, Morris and Pratt's string matcher [24].

The KMP string matcher searches for the first occurrence of a string in a text. It first preprocesses the string into a failure table, then traverses the text incrementally, searching whether the string is a prefix of the current suffix of the text. In case of character mismatch, the string is shifted farther by a distance determined by the failure table. In their original article, Knuth, Morris and Pratt display a version of the search phase that is 'compiled'-i.e., specialized - with respect to a given string [24, Section 3] [11, Appendix]. Appendix B.1] displays this specialized version in JavaScript.

A similar illustration of backward jumps can be found in Flanagan and Matsumoto's book about the Ruby programming language, where they use first-class continuations to simulate a subset of BASIC [17, Section 5.8.3]. 


\subsection{Backward and Forward Jumps}

To simulate backward and forward jumps within the same block, our translation declares a group of mutually recursive lexical variables, each denoting the continuation of a label, and uses one of these variables for each jump. Appendix B.2 shows this simulation at work with Knuth's modification of Hoare's Quicksort program [23, p. 285].

\subsection{Outward Jumps}

In modern parlance, outward jumps are exceptions. Again, our translation simulates them by declaring a lexical variable denoting the continuation of each label and using one for each jump.

Appendix B.3 displays a program that recursively descends into a binary tree, testing whether it represents a Calder mobile [13]. If one of the subtrees is unbalanced, the computation jumps out.

\subsection{Coroutines}

Whereas calling a subroutine transfers control to its beginning, calling a coroutine resumes execution at the point where the coroutine last yielded control [12]. Coroutines are useful for modeling domains with concurrent interacting processes. To account for coroutines, we use the <cmd-yield $>$ production in Figure 1 .

We have implemented a standard example of stream transducers (mapping pairs to triples [4] ), Knuth's alternative version of Quicksort using coroutines [23, p. 287], and samefringe, the prototypical example of asynchronous recursive traversals 5 , 20, 35. The samefringe program is displayed in Appendix B.4.

\subsection{Knuth's Man-or-Boy Test}

Finally, for what it is worth, our translator passes Knuth's man-or-boy test [22], using explicit thunks to implement call by name. Of course, this test exercises not jumps but recursion and non-local references. In that sense, it is more Rhino that passes Knuth's test than our homomorphic translator.

\section{Conclusion and Perspectives}

We have realized, in the modern setting of JavaScript, Landin's visionary correspondence between block-structured programs with jumps and applicative expressions 27. Our translation is idiomatic in that it maps the blocks and declarations as well as labels and jumps of the source program homomorphically to corresponding structures in the target program. The correspondence is so direct, in fact, that we can regard the image of the translation as a source language, and thus the translation as an identity function. The idiomaticity of the translation is the key to its success in producing programs that run with the same asymptotic time and space behavior as the Algol-like programs in our source language, assuming that Rhino implements downwards contargs in a reasonable way.

The simplicity and efficacy of our translation exemplifies how the principle of idiomaticity is useful for language implementation and language design: the 
correspondence between Landin's J operator and Rhino's continuation objects guides the implementation and design of our Algol-like language. In particular, we believe that a translation should preserve the control structures of source programs and map them to idiomatic control structures in the target language.

In this regard, our translation is extremely uniform and frugal in that it maps all control structures using continuation objects. Another extreme strategy, which some might consider less idiomatic and more pervasive, is to follow Steele 45] and translate all control structures using continuation-passing style or even trampolining. These translations call for JavaScript implementations that support either first-class continuations or proper tail recursion. Between these two simple extremes, there is an admittedly more complex middle path that relies on a control-flow analysis to detect particular patterns of control and translate them opportunistically to particular control-flow instructions in JavaScript that are more widely implemented well. For example:

- Programs that do not use labels and jumps can be translated without the thunks described in Section 2.2.

- Backward jumps within the same block can be translated to labeled break and continue statements.

- Backward and forward jumps within the same block can be translated using a loop that dispatches on a current state. (In the case of Quicksort 23, such a translation would reverse the 'boolean variable elimination' optimization that motivated Knuth's use of forward jumps in the first place.)

- Outward jumps can be translated using local exceptions.

In general, each control pattern should be translated idiomatically to, and thus constitutes a new use case for, a corresponding control structure in JavaScript.

Acknowledgments. This article was written while the second author was visiting Aarhus University in the fall of 2008. The authors are grateful to Dave Herman for an e-mail exchange about implementing finite-state machines in JavaScript and to Florian Loitsch and the anonymous reviewers for their comments. This work is partly supported by the Danish Natural Science Research Council, Grant no. 21-03-0545.

\section{References}

1. Kamal Abdali, S.: A lambda-calculus model of programming languages, part I: Simple constructs. Computer Languages 1(4), 287-301 (1976)

2. Kamal Abdali, S.: A lambda-calculus model of programming languages, part II: Jumps and procedures. Computer Languages 1(4), 303-320 (1976)

3. Kamal Abdali, S., Wise, D.S.: Standard, storeless semantics for ALGOL-style block structure and call-by-name. In: Melton, A.C. (ed.) MFPS 1985. LNCS, vol. 239, pp. 1-19. Springer, Heidelberg (1986)

4. Bawden, A.: Reification without evaluation. In: (Corky) Cartwright, R. (ed.) Proceedings of the 1988 ACM Conference on Lisp and Functional Programming, Snowbird, Utah, July 1988, pp. 342-351. ACM Press, New York (1988) 
5. Biernacki, D., Danvy, O., Shan, C.-c.: On the static and dynamic extents of delimited continuations. Science of Computer Programming 60(3), 274-297 (2006)

6. Bjørner, D., Jones, C.B.: Formal Specification \& Software Development. PrenticeHall International, London (1982)

7. Böhm, C., Jacopini, G.: Flow diagrams, Turing machines and languages with only two formation rules. Communications of the ACM 9(5), 366-371 (1966)

8. Burge, W.H.: Recursive Programming Techniques. Addison-Wesley, Reading (1975)

9. Burstall, R.M.: Writing search algorithms in functional form. In: Michie, D. (ed.) Machine Intelligence, vol. 5, pp. 373-385. Edinburgh University Press (1969)

10. Clements, J., Sundaram, A., Herman, D.: Implementing continuation marks in JavaScript. In: Clinger, W. (ed.) Proceedings of the 2008 ACM SIGPLAN Workshop on Scheme and Functional Programming, Victoria, British Columbia, September 2008, pp. 1-9 (2008)

11. Consel, C., Danvy, O.: Partial evaluation of pattern matching in strings. Information Processing Letters 30(2), 79-86 (1989)

12. Conway, M.E.: Design of a separable transition-diagram compiler. Communications of the ACM 6(7), 396-408 (1963)

13. Danvy, O.: Sur un exemple de Patrick Greussay. Research Report BRICS RS-04-41, DAIMI, Department of Computer Science, Aarhus University, Aarhus, Denmark (December 2004)

14. Danvy, O., Millikin, K.: A rational deconstruction of Landin's SECD machine with the J operator. Logical Methods in Computer Science 4(4:12), 1-67 (2008)

15. Kent Dybvig, R., Hieb, R.: Engines from continuations. Computer Languages 14(2), 109-123 (1989)

16. Fischer, M.J.: Lambda-calculus schemata. LISP and Symbolic Computation 6(3/4), 259-288 (1993),

http://www.brics.dk/ hosc/vol06/03-fischer.html; A preliminary version was presented at the ACM Conference on Proving Assertions about Programs, SIGPLAN Notices 7(1) (January 1972)

17. Flanagan, D., Matsumoto, Y.: The Ruby Programming Language. O'Reilly Media, Inc., Sebastopol (2008)

18. Ganz, S.E., Friedman, D.P., Wand, M.: Trampolined style. In: Lee, P. (ed.) Proceedings of the 1999 ACM SIGPLAN International Conference on Functional Programming, SIGPLAN Notices, Paris, France, vol. 34(9), pp. 18-27. ACM Press, New York (1999)

19. Girard, J.-Y.: Locus solum. Mathematical Structures in Computer Science 11(3), 301-506 (2001)

20. Hewitt, C., Bishop, P., Steiger, R., Greif, I., Smith, B., Matson, T., Hale, R.: Behavioral semantics of nonrecursive control structures. In: Robinet, B. (ed.) Programming Symposium. LNCS, vol. 19, pp. 385-407. Springer, Heidelberg (1974)

21. Jones, N.D., Gomard, C.K., Sestoft, P.: Partial Evaluation and Automatic Program Generation. Prentice-Hall International, London (1993), http://www.dina.kvl.dk/ sestoft/pebook/

22. Knuth, D.E.: Man or boy? ALGOL Bulletin 17, 7 (1964)

23. Knuth, D.E.: Structured programming with go to statements. Computing Surveys 6(4), 261-301 (1974)

24. Knuth, D.E., Morris, J.H., Pratt, V.R.: Fast pattern matching in strings. SIAM Journal on Computing 6(2), 323-350 (1977) 
25. Kranz, D., Kesley, R., Rees, J., Hudak, P., Philbin, J., Adams, N.: Orbit: An optimizing compiler for Scheme. In: Proceedings of the ACM SIGPLAN 1986 Symposium on Compiler Construction, Palo Alto, California, pp. 219-233. ACM Press, New York (1986)

26. Kranz, D.A.: ORBIT: An Optimizing Compiler for Scheme. PhD thesis, Computer Science Department, Yale University, New Haven, Connecticut (February 1988), Research Report 632

27. Landin, P.J.: A correspondence between Algol 60 and Church's lambda notation, Parts 1 and 2. Communications of the ACM 8, 89-101, 158-165 (1965)

28. Landin, P.J.: The next 700 programming languages. Communications of the ACM 9(3), 157-166 (1966)

29. Landin, P.J.: Histories of discoveries of continuations: Belles-lettres with equivocal tenses. In: Danvy, O. (ed.) Proceedings of the Second ACM SIGPLAN Workshop on Continuations, CW 1997 (1997); Technical report BRICS NS-96-13, Aarhus University, pp. 1:1-9, Paris, France (January 1997)

30. Loitsch, F.: Scheme to JavaScript Compilation. PhD thesis, Université de Nice, Nice, France (March 2009)

31. Mazurkiewicz, A.W.: Proving algorithms by tail functions. Information and Control 18, 220-226 (1971)

32. McCarthy, J.: Recursive functions of symbolic expressions and their computation by machine, part I. Communications of the ACM 3(4), 184-195 (1960)

33. McCarthy, J.: Towards a mathematical science of computation. In: Popplewell, C.M. (ed.) Information Processing 1962, Proceedings of IFIP Congress 62, pp. 21-28. North-Holland, Amsterdam (1962)

34. McCarthy, J.: A formal description of a subset of ALGOL. In: Steel, T.B. (ed.) Formal Language Description Languages for Computer Programming, pp. 1-12. North-Holland, Amsterdam (1966)

35. McCarthy, J.: Another samefringe. SIGART Newsletter 61 (February 1977)

36. McDermott, D.: An efficient environment allocation scheme in an interpreter for a lexically-scoped Lisp. In: Davis, R.E., Allen, J.R. (eds.) Conference Record of the 1980 LISP Conference, Stanford, California, August 1980, pp. 154-162 (1980)

37. Minamide, Y.: Selective tail call elimination. In: Cousot, R. (ed.) SAS 2003. LNCS, vol. 2694, pp. 153-170. Springer, Heidelberg (2003)

38. Moggi, E.: Notions of computation and monads. Information and Computation 93, 55-92 (1991)

39. Lockwood Morris, F.: Correctness of Translations of Programming Languages - an Algebraic Approach. PhD thesis, Computer Science Department, Stanford University (August 1972), Technical report STAN-CS-72-303

40. Lockwood Morris, F.: The next 700 formal language descriptions. Lisp and Symbolic Computation 6(3/4), 249-258 (1993); Reprinted from a manuscript dated (1970)

41. Reynolds, J.C.: Definitional interpreters for higher-order programming languages. In: Proceedings of 25th ACM National Conference, Boston, Massachusetts, pp. 717-740 (1972); Reprinted in Higher-Order and Symbolic Computation 11(4), 363397 (1998), with a foreword [42]

42. Reynolds, J.C.: Definitional interpreters revisited. Higher-Order and Symbolic Computation 11(4), 355-361 (1998)

43. Schinz, M., Odersky, M.: Tail call elimination on the Java Virtual Machine. In: Benton, N., Kennedy, A. (eds.) BABEL 2001: First International Workshop on MultiLanguage Infrastructure and Interoperability, Firenze, Italy, September 2001. Electronic Notes in Theoretical Computer Science, vol. (59), pp. 155-168. Elsevier Science, Amsterdam (2001) 
44. Stallman, R.M.: Phantom stacks: If you look too hard, they aren't there. In: AI Memo 556, Artificial Intelligence Laboratory, Massachusetts Institute of Technology, Cambridge, Massachusetts (July 1980)

45. Steele Jr., G.L.: Rabbit: A compiler for Scheme. Master's thesis, Artificial Intelligence Laboratory. Massachusetts Institute of Technology, Cambridge, Massachusetts, Technical report AI-TR-474 (May 1978)

46. Talcott, C.L.: The Essence of $\mathcal{R}$ um: A Theory of the Intensional and Extensional Aspects of Lisp-type Computation. PhD thesis, Department of Computer Science, Stanford University, Stanford, California (August 1985)

47. Tarditi, D., Lee, P., Acharya, A.: No assembly required: Compiling Standard ML to C. ACM Letters on Programming Languages and Systems 1(2), 161-177 (1992)

48. Thielecke, H.: Comparing control constructs by double-barrelled CPS. HigherOrder and Symbolic Computation 15(2/3), 141-160 (2002)

49. Wadler, P.: The essence of functional programming (invited talk). In: Appel, A.W. (ed.) Proceedings of the Nineteenth Annual ACM Symposium on Principles of Programming Languages, Albuquerque, New Mexico, pp. 1-14. ACM Press, New York (1992)

\section{A Grammar of JavaScript in the Image of the Translation}

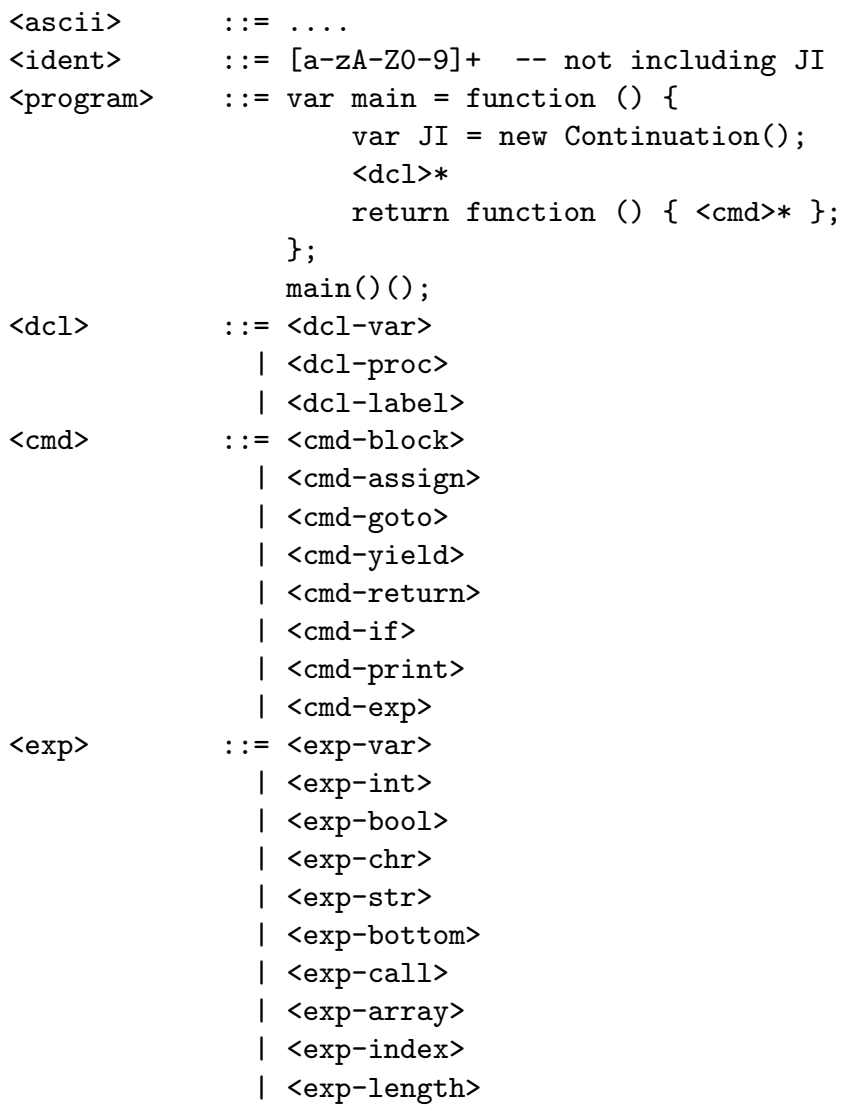




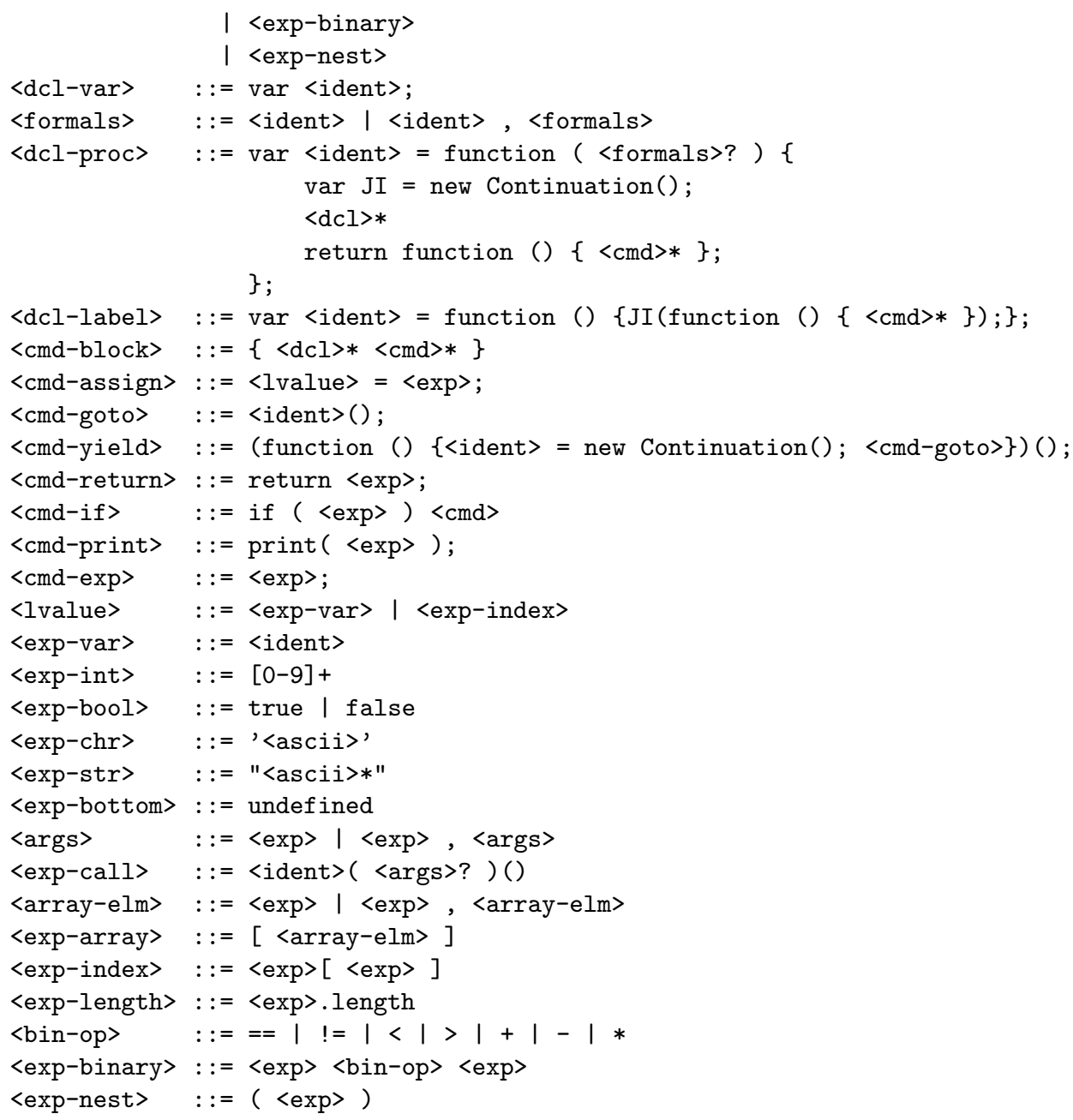

\section{B Example JavaScript Programs}

In the following examples we represent trees in terms of arrays. Depending on the use, a tree can have either weighted internal nodes, as used in Calder mobiles (Appendix B.3), or weighted leaf nodes, as used in Samefringe (Appendix B.4).
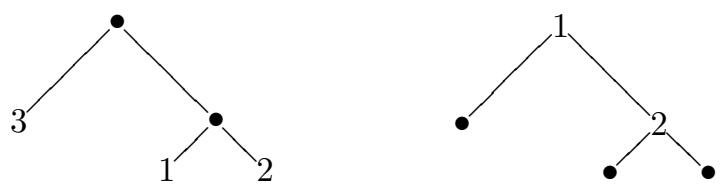
In each case we denote the weightless nodes $(\bullet)$ with -1 . A tree is then encoded as a linear block of values with the successor functions left $(n)=2 n+1$ and $\operatorname{right}(n)=2 n+2$ as illustrated below.

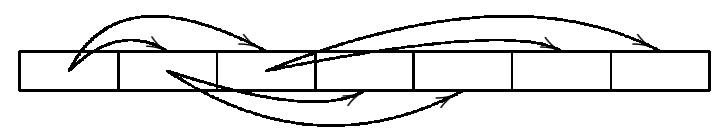

\section{B.1 Backward Jumps: KMP String Search}

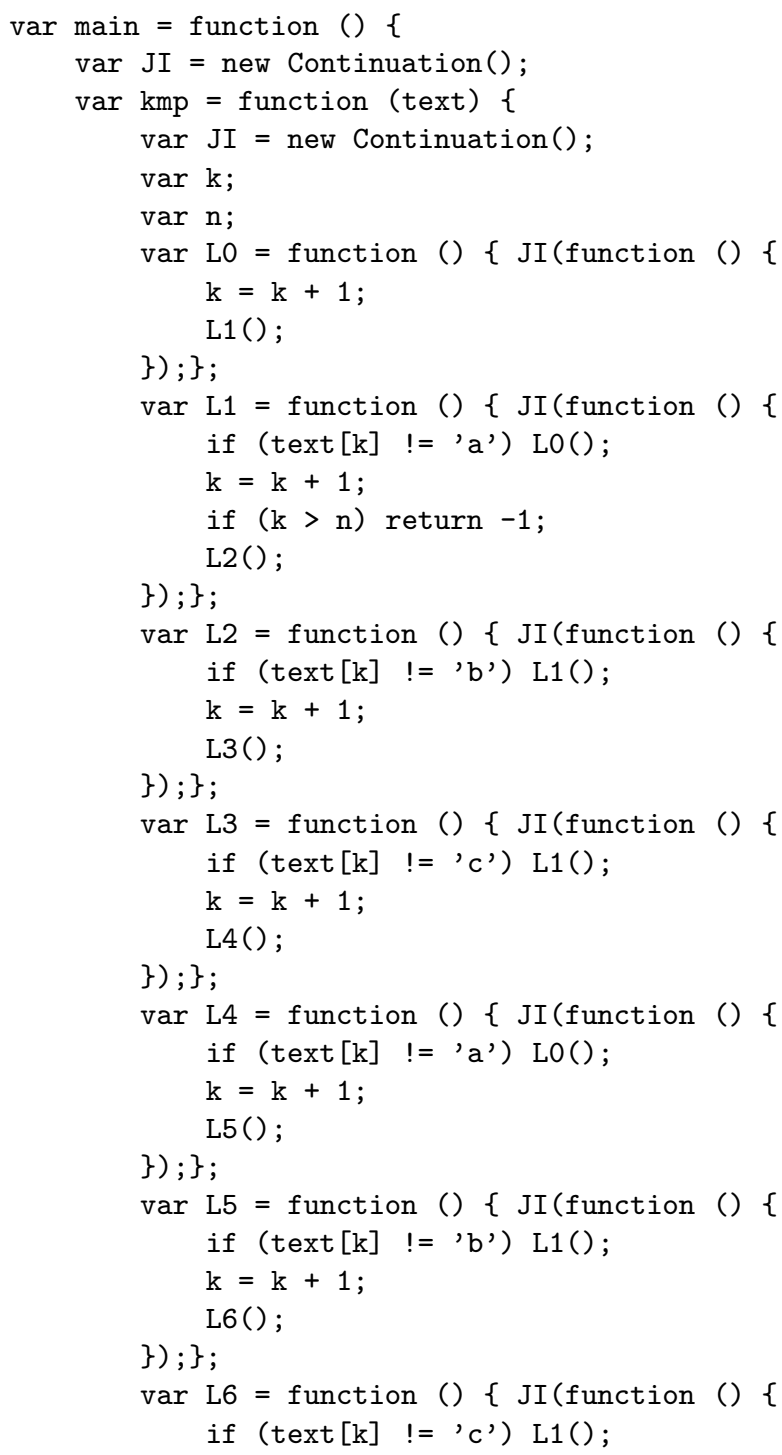




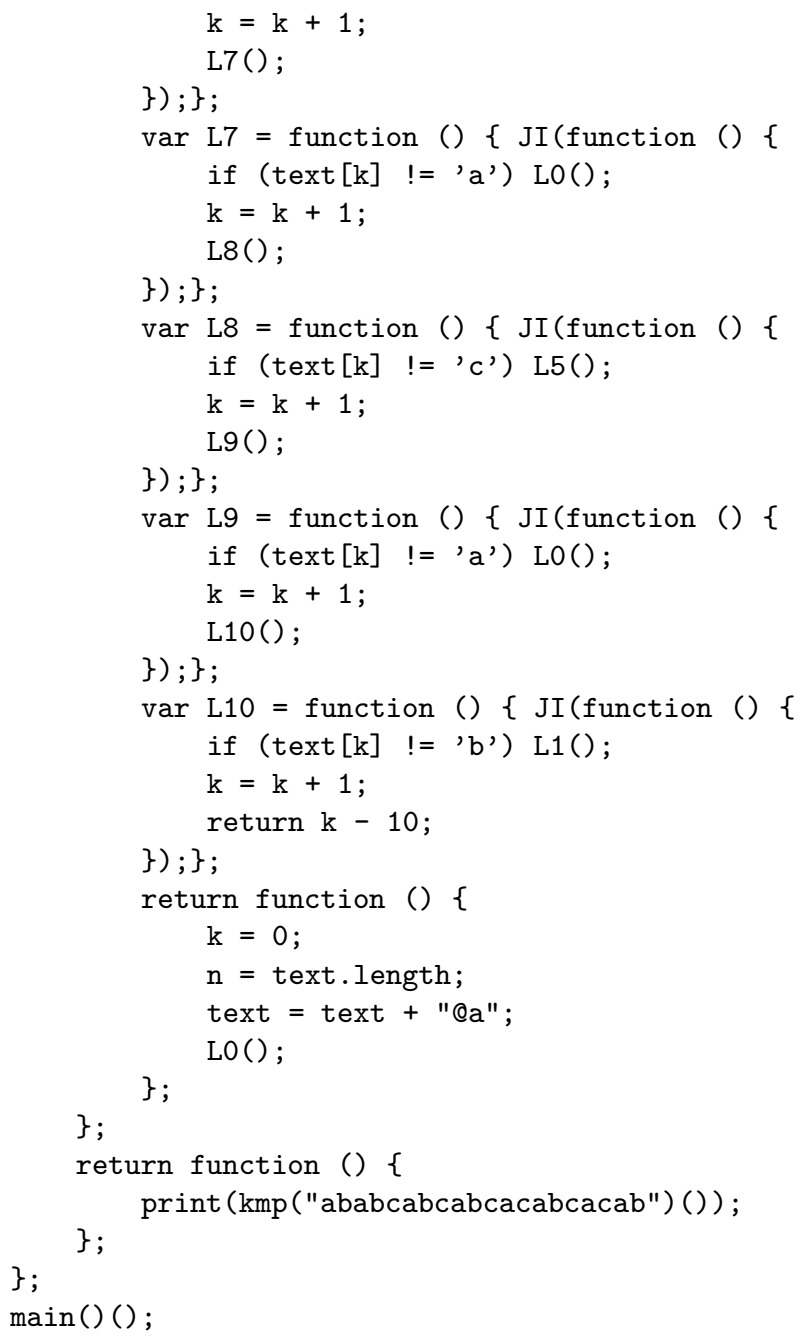

\section{B.2 Backward and Forward Jumps: Quicksort}

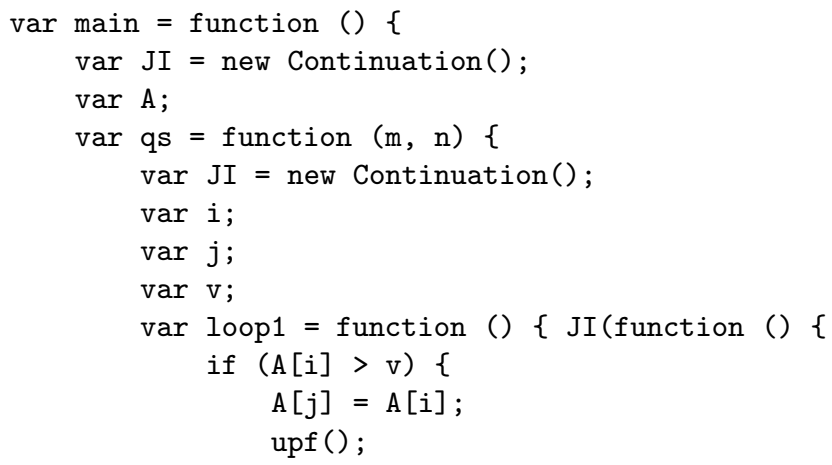




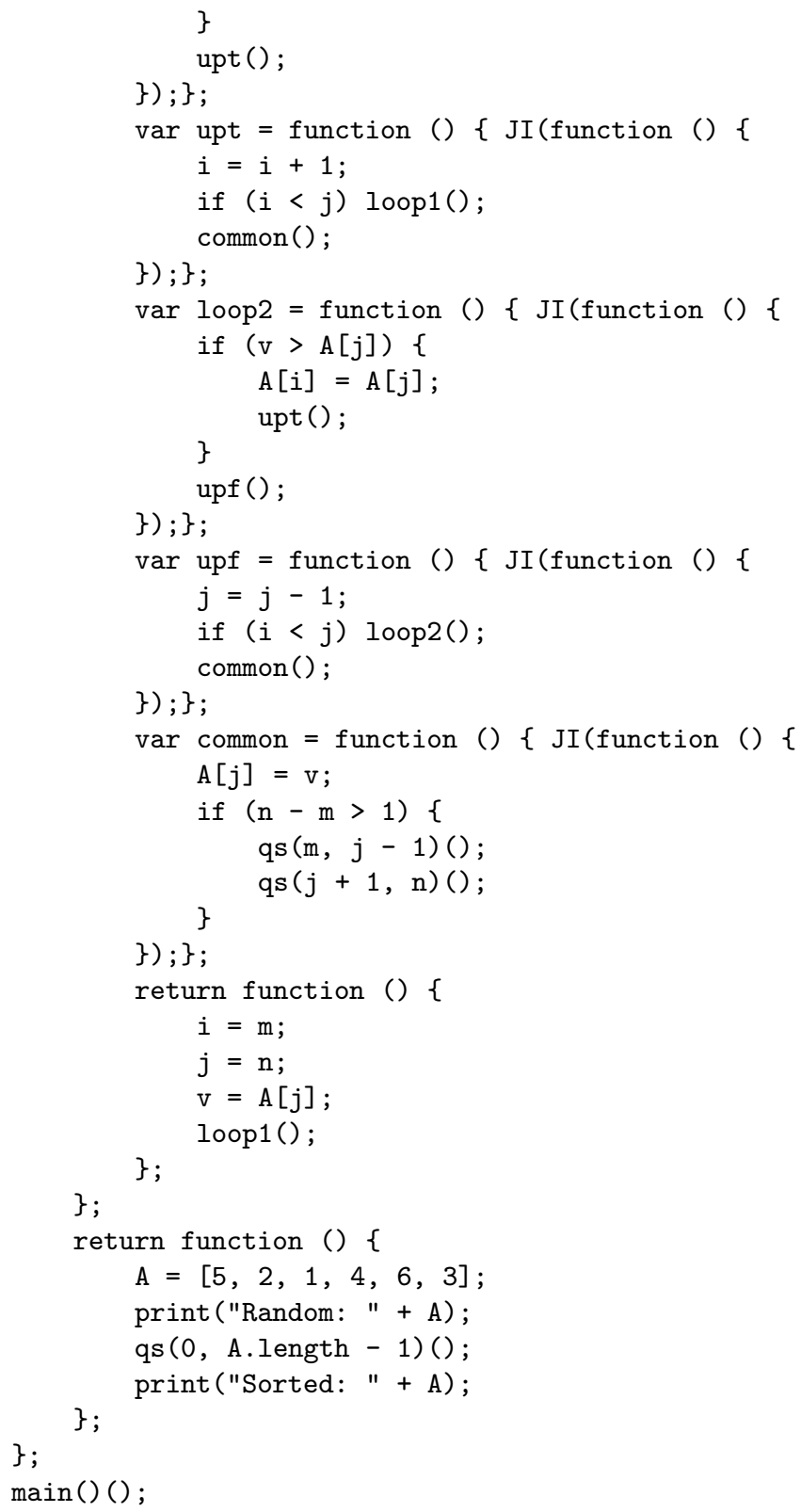

\section{B.3 Outward Jumps: Calder Mobiles}

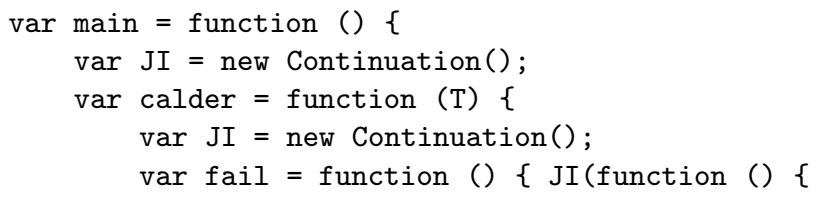




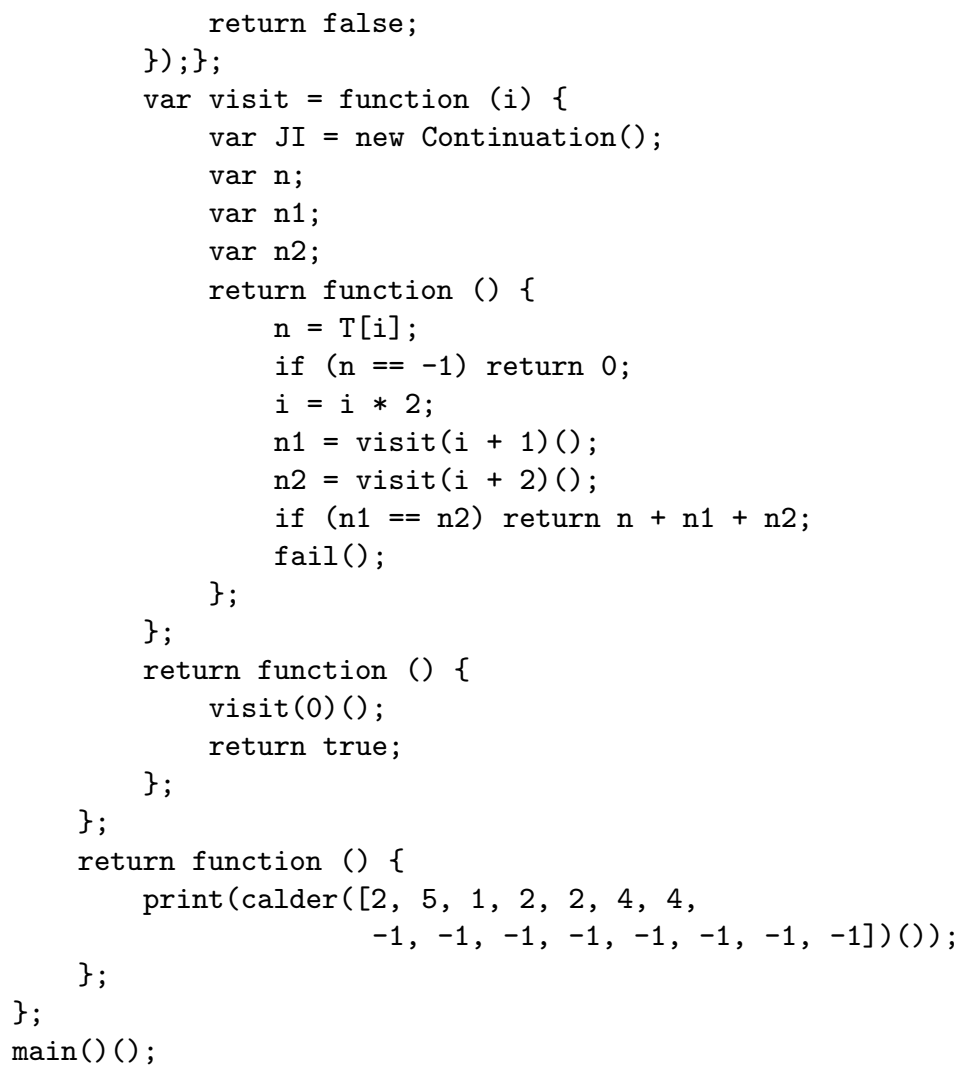

\section{B.4 Coroutines: Samefringe}

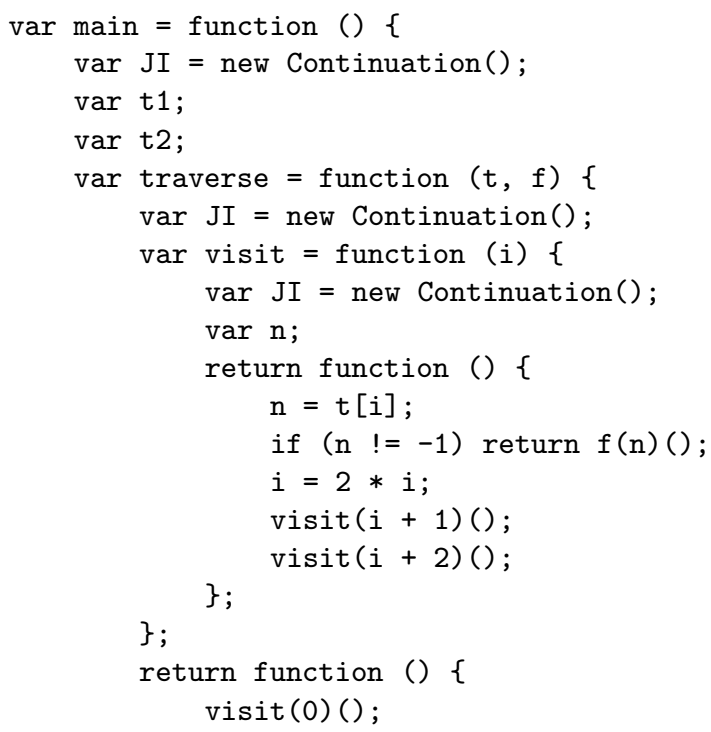




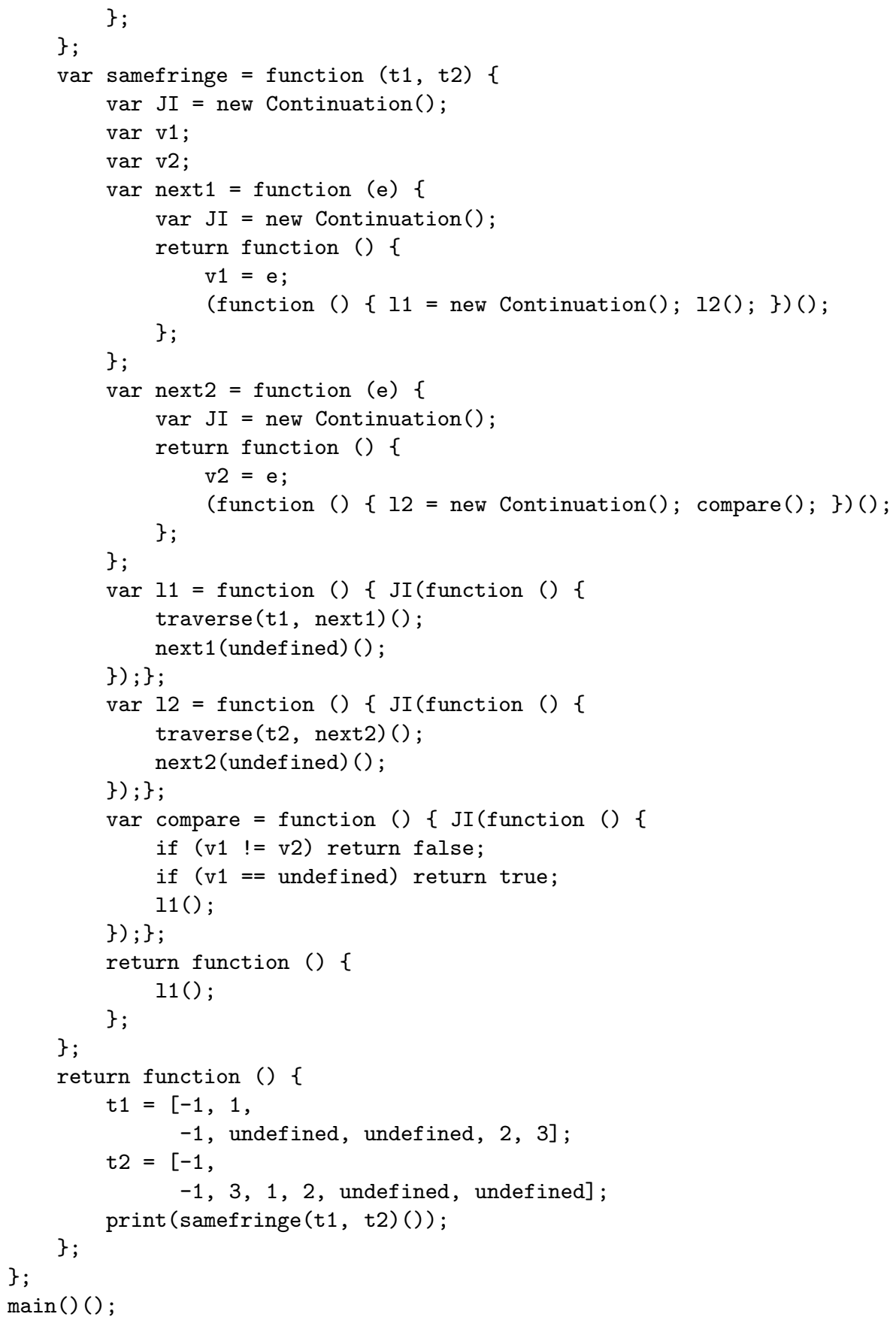

